\title{
The Use of Optogenetic and DREADDS Techniques: Applications to the Behavioral Pathology in Parkinson's Disease (Review
}

DOI: $10,17691 / \mathrm{stm} 2019.11,2.21$

Received January 24, 2019

N.1. Novikov, DSc, Researcher, Laboratory of Systemic Organization of Neurons;

E.S. Brazhnik, PhD, Researcher, Laboratory of Systemic Organization of Neurons; V.F. Kichigina, DSc, Chief Researcher, Laboratory of Systemic Organization of Neurons

Institute of Theoretical and Experimental Biophysics, Russian Academy of Sciences, 3 Institutskaya St.,

Pushchino, Moscow Region, 142290, Russia

Recent advances in genetics have led to the development of novel optogenetic and chemogenetic tools that allow selective and remote interrogation of neural circuits using light-sensitive opsins and engineered G-protein-coupled receptors activated by inert druglike small molecules. These novel techniques have been rapidly applied to many aspects of neuroscience, including research on learning and memory, decision making, and goal-directed behavior. By using specific light-sensitive opsins and DREADDs (designer receptors exclusively activated by designer drugs) to monitor the electrophysiological, biochemical, and behavioral outputs of specific neuronal types, the links between brain activity and behavior can be better evaluated. Additionally, optogenetics and DREADDs are beneficial in studying the pathogenesis of neurological conditions, such as depression, anxiety, pain, drug addiction, as well as neurodegenerative diseases, and may ultimately have therapeutic potential.

Key word: Parkinson's disease; movement disorders; basal ganglia; dopamine; light-sensitive ion channels; modified G-proteincoupled receptors.

\section{Introduction}

Over the past decade, optogenetic and chemogenetic tools have enabled detailed mapping of functional neuronal circuits and pathways necessary for normal sensory, motor, and cognitive function. Understanding the network architecture at the level of both local microcircuits and distributed macrocircuits may facilitate the development of new therapeutic strategies for the treatment of various neurological disorders. In this review, we present examples of research applications of optogenetics and chemogenetics to uncover the specific neurological function of the basal ganglia-thalamocortical motor circuits in health and disease. Specifically, we discuss their contribution to the understanding of motor symptomology in Parkinson's disease induced by progressive loss of midbrain dopaminergic nigral neurons and ultimate development of exaggerated involuntary behavior (dyskinesias) as a result of dopamine replacements therapy. The comparative strengths and weaknesses of these approaches are reviewed for both preclinical and translational applications.

\section{Parkinson's disease, basal ganglia circuitry, and dopamine}

Parkinson's disease and motor dysfunction. Parkinson's disease (PD) is a complex and highly variable neurodegenerative movement disorder [1]. The classical motor symptoms of PD (akinesia, bradykinesia, rigidity, tremor, and postural abnormalities) are associated with the loss of dopaminergic neurons in the substantia nigra pars compacta (SNc) of the midbrain and a decline in the caudate-putamen dopamine content. The loss of dopaminergic inputs to the striatum has been implicated in the induction of basal ganglia (BG) circuitry malfunction in PD. The BG is the primary brain system affected by PD. BG includes a complex network of nuclei in the forebrain, which play critical role in motor control. It has been suggested that disorganization of the BG may be closely related to various neurodegenerative diseases, such as PD [2]. The BG complex, which receives inputs from the cerebral cortex and thalamus and projects downstream to motor regions that control movement, participates in the processing of information from the neocortex through several local circuits to remote regions, such as superior colliculus, motor thalamus, cerebellum, and brainstem pedunculopontine nucleus. All of these regions are essentially implicated in the control of locomotion [3-5].

Dopamine is one of the critical neurotransmitters and neuromodulators in the BG circuitry affecting the control of motor function [4-6]. Dopaminergic neurons of the SNc innervate medium spiny GABAergic projection neurons (MSNs) in the striatum [7]. The BG circuitry receives information from cortical regions and processes

Corresponding author: Nikolay I. Novikov, e-mail: nikolay_novikov@hotmail.com 
it through parallel and distinct neural circuits connecting the two BG input regions, the subthalamic nucleus (STN, through the hyperdirect pathway) and striatum, to the BG output nuclei. Principal projection GABAergic neurons in striatum form two separate pathways. The direct (striatonigral) pathway is composed of GABAergic medium spiny neurons (dMSNs) that predominantly express dopamine D1 receptors (D1Rs) and project monosynaptically to the BG output nuclei, globus pallidus interna (GPi) and substantia nigra pars reticulata $(\mathrm{SNr})$. In contrast, the indirect (striatopallidal) pathway predominantly expresses D2 receptors (D2Rs) and projects to the globus pallidus externa (GPe), which then relays to the $B G$ output nuclei $(\mathrm{GPi} / \mathrm{SNr})$. These two pathways are thought to create a dynamic balance exerting opposing actions on the control of movement, cognition, and motivational processes (for review see [5, 8]). Accordingly, an imbalance between these pathways has been postulated for several neurological disorders, including PD [9, 10].

Treatment of motor symptoms in Parkinson's disease. Currently, no treatment has been demonstrated to terminate or even slow the progression of PD. Pharmacological therapy with 3,4-dihydroxyphenyl-Lalanine (L-DOPA) considerably alleviates the severe motor symptoms seen in PD patients [11, 12]. Although initially very effective, motor complications in the form of L-DOPA-induced dyskinesia (LID) typically occur within a few years of starting treatment [13].

In the last decade, deep brain stimulation (DBS) has been implicated as an effective treatment to ameliorate PD symptoms by stimulating a part of the BG circuitry at a high frequency (approx. $120 \mathrm{~Hz}$ ). While DBS is valuable tool in the treatment of PD motor symptoms, the precise mechanism of its action has remained unclear. Recent therapeutic innovations for the treatment for PD include (a) cell transplantation therapy with human embryonic stem cells, (b) human induced pluripotent stem cells and human fetal mesencephalic tissue, (c) immunological and anti-inflammatory therapy using antibodies, (d) gene therapy with AADC$\mathrm{TH}-\mathrm{GCH}$, (e) viral vector-mediated gene delivery, (f) RNA interference-based therapy, (g) CRISPRCas9 gene editing system, and alternative methods such as optogenetics and chemogenetics. Although these methods currently have a series of challenges, they seem to be promising techniques for Parkinson's treatment in the future [14].

Animal models of Parkinson's disease. Animal models of PD have been widely used in the past four decades to investigate the pathophysiology of this neurodegenerative disorder and to evaluate novel therapies for motor dysfunction and LID. These models have been classically based on the systemic or local (intracerebral) administration of neurotoxins, such as 1-methyl-4-phenyl-1,2,3,6-tetrahydropyridine (MPTP) or 6-hydroxydopamine (6-OHDA), which are able to replicate most of the pathological and phenotypic features of PD in mammals (i.e. rodents or primates). In the last ten years, the 'genetic era' of PD has significantly expanded the research possibilities in this field, with the development of various transgenic animal models that include most of the disease-causing mutations identified for monogenic forms of familial PD. Both toxin-induced and transgenic classes of animal PD models have their own advantages and limitations (for review see [15]).

Excessive synchronization in the motor circuits. A hallmark of $B G$ dysfunction in $P D$ is the amplification of synchronous, rhythmic activity, particularly in the beta frequency range $(13-30 \mathrm{~Hz})$ [16-21]. Exaggerated $B G$ beta activity is commonly found in patients with $P D$, and the amplitude of such activity has been linked to motor impairment and dopaminergic tone [18]. Although some clinical data and results from animal models suggest that exaggerated beta synchronization in the basal ganglia-thalamocortical circuits does not cause motor symptoms in PD [22-24], its reduction with dopamine replacement therapy and DBS of the STN is correlated with symptomatic improvement [25-30]. Therefore, understanding how beta oscillations originate and propagate and how they can be diminished could be of potential therapeutic value.

Transient pharmacological manipulations, targeted surgical ablation, and excitotoxic lesions of selective BG nuclei, along with a variety of methods for recording neural activity, have provided critical insight into the network organization that supports the development of motor symptoms and circular synchrony in the PD patient and animal models of PD. However, while these approaches are powerful, they are limited in terms of neuronal specificity and temporal and spatial resolution. The complex relationship between the specific cell types and neurotransmission in various nodes of motor circuits has been difficult to thoroughly characterize. The introduction of recent advances in chemogenetic and optogenetic methods in neuroscience research [31, 32] over the past decade has enabled access cell-type specificity, real-time bidirectional control of neuronal activity, and the ability to manipulate circuits in a projection-pathway-specific manner.

\section{Optogenetic and chemogenetic methods in neuroscience}

Modulation of neuronal activity has played a key role in neuroscience and behavioral research, complementing electrophysiological and anatomical experiments to gain insight into the functional roles of specific brain regions, circuits, and cells. Optogenetics, chemogenetics, and novel designer receptors have revolutionized the way neuroscientists can interrogate neural circuits. These new tools are being rapidly applied to many aspects of neuroscience, including the study of PD circuitry and therapies [33]. Genetically encoded tools designed for acute and chronic manipulation of circuit function are now commonly used to assess the contribution of cell 
type-specific populations and individual neural circuit components to animal behavior [34].

Optogenetic techniques. Optogenetic methods use targeted expression of light-responsive proteins (opsins) in specific neurons to manipulate their activity [35]. Optogenetics allows identification of the functions of individual parts of the brain by rapidly altering their activity, whilst minimizing the likelihood that the brain will compensate for these changes. Many different opsins are available for neuroscientific applications, including ion channels and pumps that allow depolarization or hyperpolarization of cell membranes, and consequent excitation or inhibition of neuronal activity, respectively. The most common excitatory tools (i.e., depolarizing opsins) include variants of the cation channel channelrhodopsin (ChR) [32, 36], while commonly used hyperpolarizing opsins are proton or chloride pumps, such as archaerodopsin (Arch) [37] and halorohodopsin (NpHR) [38]. When illuminated with the appropriate wavelength (blue, yellow, green, orange and red light), these opsins selectively respond with millisecond time courses, allowing precise temporal control of neuronal activity with rapid onset and offset of excitation or silencing of neuronal activity. This technique requires implantation of optic fibers into the brain region containing transfected cells or into the target region with axonal terminals from the afferent sites.

Chemogenetic techniques. Chemogenetic tools involve the activation of engineered G-protein-coupled receptors that are expressed on target neurons and can then be activated by systemic administration of biologically inert drugs $[39,40]$. The most widely used chemogenetic technique relies on the expression of designer receptors exclusively activated by designer drugs (DREADDs) in transfected neurons. In order to determine whether specific neurons drive a particular behavior, DREADDs can be expressed by intracerebral microinjection into selected cell populations. Two groups of selective designer receptors have been generated: synthetic variants of the human muscarinic acetylcholine receptors coupled to $\mathrm{Gi} / \mathrm{o}, \mathrm{Gq} / 11$, or $\mathrm{Gs}$ proteins that are activated by clozapine-N-oxide (CNO) and a novel inhibitory designer receptor developed from the kappa opioid receptor (KORD) that responds to a different inert ligand, salvinorin B (SalB) [41]. With two different DREADDs, responding to different exogenous ligands, it is possible to control the neuronal activity in a bidirectional way: exciting a particular neuronal population and then rapidly inhibiting it to provide conclusive evidence of its involvement in a specific behavior. The DREADD technique, developed by Roth and colleagues [42-45], has emerged as a powerful tool in neuroscience for selective manipulation of cellular activity and neuronal circuits. The DREADD method allows for manipulation of brain circuits and neuron populations that are altered under numerous pathological conditions and, thus, has been found to be beneficial in biomedical research. Chemogenetic agents such as DREADDs have several advantages over other pharmacological and nonpharmacological treatment modalities, such as targeted lesions and DBS. Chemogenetic-based treatment directly increases or suppress neuronal excitability and is reversible, much less invasive, and better controlled (by adjusting CNO or SalB dosage) than the above modalities. Although chemogenetic-based treatment requires stereotactic injection of the DREADDs-expressing viral vectors through a small burr hole, it is less invasive than optogenetic stimulation, as it does not require a light source to reach the target neurons.

Both optogenetic and DREADD methodologies rely on the expression of light-sensitive opsins/designer receptors that can be achieved by: (1) viral transduction (e.g. via promoter-specific adeno-associated viruses (AAV)), (2) animal transgenesis (for example, transgenic mice expressing receptors under the TET-off system), or (3) a combination of both (for instance, injection of viral vectors with the gene reversed and floxed by two pairs of lox sites (DIO; double-floxed inverse orientation)) into Cre recombinase driver animal lines. The common aspects of facilitating cell-type- and pathway-specific activation or silencing of neurons have been reviewed in detail elsewhere [34, 46-48].

In summary, the novel optogenetic and DREADD tools have enabled the functional and behavioral dissection of many discrete brain circuits with exquisite precision [49-51]. Moreover, they can potentially be used to enhance existing treatments and generate novel therapeutic strategies for neurodegenerative disorders, including PD.

\section{Optogenetic and DREADD tools to dissect motor circuits in animal models of Parkinson's disease}

Our current knowledge of BG circuits and functions is strongly influenced by recent advances accomplished using optogenetic and chemogenetic methods. These techniques have been used to uncover the contributions of the direct and indirect pathways to movement and reinforcement [52-60], provide new descriptions of neuronal heterogeneity within the BG [61-63], explain the functional roles of dopamine depletion in striatal neurons [64-66], and provide new insights into the changes in the plasticity of BG circuits in pathological conditions [67-69]. However, advances adopting the use of opto- and chemogenetic tools have been achieved, primarily, in rodent models, while the use of these techniques in non-human primates has remained limited.

Motor behavior depends on coordinated activity across multiple brain regions. Within such networks, the highly connected hub regions are expected to disproportionally influence motor behavioral output; therefore, malfunctioning of neuronal and circuit activity due to progressive degeneration of nigral dopamine 
neurons could be implemented in the induction motor dysfunction in PD.

Impact of the basal ganglia circuits in induction of motor symptoms in Parkinson's disease. The generation of movement depends on the integration of vast amounts of information in the striatum, the major input nucleus of the BG. Changes in information processing at the striatal level are assumed to impact the activity of downstream BG nuclei, which in turn influence brain-wide networks, including the motor thalamus, motor cortex, and brainstem inputs. The striatum is comprised of two intermixed classes of GABAergic projection neurons that exert opposing effects on downstream structures. The direct-pathway medium spiny projection neurons (dMSNs) express D1 dopamine receptors and project directly to the $\mathrm{SNr}$, the main output nucleus of the BG. The indirect-pathway projection neurons (iMSNs) express D2 receptors and project to the $\mathrm{SNr}$ indirectly via the globus pallidus external segment $(\mathrm{GPe})$. These circuits are believed to have opposing effects on movement: iMSNs, whose activation promotes movement suppression, become hyperactive, whereas dMSNs, whose activation promotes movement initiation, become hypoactive in PD state [70-73]. Thus, a relative imbalance in the activity of the two pathways is thought to play a central role in the induction of hypokinetic symptoms in PD.

Experimental validation of this classical model in awake behaving animals was not possible with the use of traditional methods. The most conclusive evidence for motor antagonism between these pathways has come from rodent optogenetic studies, which showed increased and reduced locomotion during selective optogenetic stimulation of the direct and indirect pathway, respectively, in BAC transgenic mice selectively expressing ChR2 in dopamine D1, D2, or adenosine A2A receptor-expressing striatal projection neurons $[53,55]$. Kravitz et al. [55] demonstrated restoration of normal motor function in mice with bilateral 6-OHDA lesions by optogenetic bilateral stimulation of dorsomedial striatal direct pathway neurons (dMSNs). At the neuronal level, Ryan et al. [74] provided evidence that chronic dopamine loss bidirectionally affects the firing of direct and indirect pathway neurons in the striatum. Using optogenetically labeled striatal single-unit recordings in dopamine-depleted mice, the authors evaluated circuit dysfunction in parkinsonism and found that following dopamine depletion, iMSN firing was elevated, while dMSN firing was dramatically and persistently reduced. These findings provided key insights into the circuit mechanisms underlying parkinsonism with possible implications for developing targeted therapies for PD. However, other studies that used a series of statedependent optogenetic manipulations to examine the activity of these projection pathways during the initiation of natural movements or learned action sequences have revealed that both the direct and the indirect striatal pathways are concurrently active during sequence initiation [52, 59, 75-77]. Moreover, the two pathways regulate movements by acting in a complementary supportive and permissive manner, rather than through opposing hyperkinetic and akinetic effects [60, 77].

Taken together, these findings suggest that novel interventions that preferentially recruit the striatal direct pathway may represent a possible therapeutic strategy for the treatment of motor symptoms in PD.

Striatal cholinergic interneurons. The ability of nicotinic and muscarinic cholinergic receptors to regulate striatal dopamine release makes the cholinergic system a critical player in modulation of the output of D1 and D2 striatal MSNs and BG function. Cholinergic interneurons (CINs) represent only $1-2 \%$ of striatal neurons, but they densely innervate the principal striatal MSNs. CINs control and integrate multiple striatal synaptic inputs and outputs to regulate motor activity under normal physiological conditions. However, the complicated balance between dopaminergic and cholinergic neurotransmission in the striatum has been difficult to characterize with traditional methods. In PD, the loss of innervation of nigral dopaminergic neurons in the striatum leads to alteration in striatal cholinergic signaling and increased striatal CINs excitability [78]. In a recent study that combined optogenetics with behavioral and electrophysiological approaches in transgenic ChATCre mice, Maurice et al. [79] showed that optogenetic inhibition of CINs reduces parkinsonian motor signs in PD mouse models, providing direct demonstration of their role in parkinsonian motor dysfunction. At the neuronal level, this study provides evidence that inhibition of striatal CINs in parkinsonian mice differentially impacts the excitability of striatal D1 and D2 MSNs, enhances cortically evoked inhibition in SNr, normalizes pathological bursting activity in the main BG output nucleus, and potentiates the direct striatonigral pathway in cortical information processing. By contrast, CIN inhibition in nonlesioned mice does not affect locomotor activity and does not modify spontaneous or cortically driven activity in the BG output, suggesting that the role of these interneurons in motor function is highly dependent on dopamine tone.

Globus pallidus externa. The external globus pallidus $(\mathrm{GPe})$ is a key contributor to motor suppressing pathways in the BG, although the neuronal heterogeneity of this BG nucleus has long remained an unexploited resource for therapeutic interventions. Dopamine deficiency in PD is suggested to shift the balance between the BG pathways, enhancing the activity of the striatal indirect pathway to the GPe; therefore, the activity of the inhibitory GPe nucleus is expected to be largely decreased in the parkinsonian state [80]. Remarkably, the study of Mastro et al. [81] demonstrated that optogenetic interventions that dissociate the activity of two neuronal populations in the GPe, elevating the activity of parvalbumin-expressing neurons (PV-GPe, projecting to the STN and SNr) over that of neurons expressing Lhx6 (Lhx6-GPe, projecting back to the 
striatum), induces long-lasting recovery of movement in dopamine-depleted mice and attenuates pathological activity in the BG circuit, despite the permanent loss of dopamine. Additionally, selective optogenetic inhibition of Lhx6-GPe neurons substantially restores movement in dopamine-depleted mice. In contrast, cell-type nonspecific optogenetic excitation of the GPe does not improve movement in dopamine-depleted mice. These results establish the behavioral relevance of functionally distinct neuronal subpopulations in the GPe and suggest their potential as therapeutic nodes for the long-term restoration of movement in PD. A recent study by Assaf and Schiller [82] reported that enhancement of total activity in the GPe nucleus with excitatory DREADDs was sufficient to considerably improve all aspects of motor behavior in both hemiparkinsonian and bilaterally dopamine-lesioned mice.

Basal ganglia output. Hypokinetic disorders, such as PD, are hypothesized to result from a complex series of changes in the activity of striatal projection neuron subpopulations resulting in an increase in BG output (GPi/SNr). Specifically, dopamine deficiency in PD is expected to shift the balance between the BG pathways, enhancing the activity of the indirect and reducing the activity of the direct pathway, which, in turn, is expected to enhance the activity of the inhibitory output nuclei, GPi and SNr, and reduce the cortical drive in the BGthalamocortical circuit [83-85]. Using a chemogenetic approach to express inhibitory DREADDs in the GPi/SNr and various motor behavioral tasks in 6-OHDA-induced parkinsonian mice, Assaf and Schiller [82] were able to show significant improvement in motor performance by suppressing the activity of the BG output nuclei. Another study [86] reported similar improvement in motor behavior in hemiparkinsonian rats using optogenetic approaches. The authors showed that optogenetic inhibition of activity in the entopeduncular nucleus, an analog of the primate $\mathrm{GPi}$, in the hemiparkinsonian rat increased thalamic output and improved parkinsonian behavior (contralateral forelimb akinesia), while optogenetic excitation of the entopeduncular nucleus did not have an effect.

As already noted, the BG orchestrate complex motor behavior, including action selection, patterning, and coordination, by modulating downstream motor areas such as the motor thalamus, motor cortex, and brainstem inputs. Striatal MSNs, modulated by dopamine, receive excitatory inputs from several brain regions, including the cortex, thalamus, and feedback from brainstem motor regions. The striatum is thought to control movement, integrating dopaminergic and glutamatergic inputs from sensorimotor cortical regions and motor thalamus [87-89].

Cortex and impact of cortico-subthalamic pathway. Functional deficits in cortical circuits are thought to be implicated in a number of neurodegenerative motor disorders [90, 91]. The motor cortex is one of the final cortical outputs to downstream spinal motor neurons, and it is fundamental for controlling voluntary movements [92, 93]. The motor cortex is widely credited with expanding the behavioral repertoire of mammals by enabling the acquisition and execution of new motor skills, but its specific contributions have been difficult to identify.

Coordinated motor behaviors involve performance of sequential patterns of distinct movements in a specific order, which is thought to require synaptic plasticity within corticostriatal circuits that route information through the BG. Rothwell et al. [94] used genetically and anatomically targeted manipulations of specific circuit elements in mice to isolate the source and target of a corticostriatal synapse, thereby demonstrating that serial order performance is regulated by a monosynaptic pathway linking the secondary motor cortex (M2) to striatal cells that form the direct pathway (dMSNs) through the BG. These monosynaptic connections between M2 and dMSNs are selectively strengthened by experience-dependent and serial order learning, shifting the balance of striatal output in a manner that is critical for performing a behavioral routine.

Based on the classical model of BG function in PD, the motor cortex is thought to be hypoactive in PD [9, 71, 84, 95]. Despite the hypothesized importance of this model, however, it has been difficult to definitively demonstrate which properties of cortical function are disrupted in PD. As mentioned above, abnormal activity patterns in the motor circuit are correlated with the appearance of parkinsonism. Evidence from multiple studies has suggested that the cortico-subthalamic hyperdirect pathway plays a key role in patterning pathological activity in the STN in PD. The motor symptoms of PD are linked to abnormally correlated and coherent activity in the cortex and STN [96-101]. However, the results from MPTP-treated parkinsonian monkeys provide strong evidence for a partial loss of the hyperdirect corticosubthalamic projection in PD [102]. Consistent with this, cortico-STN transmission strength was found to be diminished by $50-75 \%$ in parkinsonian mice through the loss of axo-dendritic and axo-spinous synapses and was incapable of long-term potentiation and less effective at patterning the STN activity. Optogenetic, chemogenetic, genetic, and pharmacological interrogations have all suggested that downregulation of cortico-STN transmission in PD mice was induced by increased striatopallidal transmission, leading to disinhibition of the STN and increased activation of STN NMDA receptors. Together, these studies suggest that loss of dopamine generates a maladaptive shift in the balance of synaptic excitation and inhibition in the STN, which contributes to parkinsonian activity and motor dysfunction [103].

Motor thalamus. The motor thalamus is considered to have an important role in the circuitry that generates movement disorders. Indeed, bradykinesia and hypokinesia in PD have been attributed to increased activity in BG output nuclei and subsequent reduction in the thalamocortical excitatory drive to motor and 
premotor areas [9, 71, 84, 95]. The mechanisms of pathological functioning of the motor thalamus in PD remains unclear. Increased inhibition of thalamic neurons is capable of generating low-threshold bursts through the activation of T-type $\mathrm{Ca}^{2+}$ channels, thereby inducing a strong post-synaptic motor cortex response. A recent study by Kim and Kim [104] employed an optogenetic approach to control the activity of specific inhibitory inputs to the ventrolateral thalamus, and showed that increase in rebound burst firing in the ventrolateral thalamic neurons can trigger PD-like motor abnormalities in wild-type mice, while reduction of rebound excitability of thalamic neurons rescues normal motor function in dopamine-depleted mice. These findings suggest that an inhibitory synaptic mechanism mediates motor dysfunction by generation of rebound excitability in the thalamocortical pathway and possible recruitment of abnormal muscle tension, which may lead to whole-body rigidity and tremor.

Among other motor thalamic nuclei, the CM/Pf complex, the caudal intralaminar region of the thalamus, receives inhibitory input from BG and provides the main source of thalamic inputs to the striatum and STN [105107]. Evidence of extensive cell loss in this nucleus $[108,109]$ and therapeutic antiparkinsonian effect of DBS of the CM/PF in PD patients and PD rats [110, 111], as well as the reported hyperactivity of thalamic Pf neurons in 6-OHDA-lesioned rats [112], have implicated this nucleus in the pathophysiology of parkinsonism $[113,114]$. Thalamostriatal axon terminals of CM/ Pf origin form axodendritic synapses with both direct and indirect striatal MSNs and predominantly with the cholinergic interneurons (for review see [114]). Alteration of synaptic plasticity at corticostriatal terminals has been suggested to contribute to changes in striatal neuron activity in PD. Using opto- and chemogenetic methods, Parker et al. [115] reported that striatal dopamine depletion in PD mouse models altered the synaptic strength of thalamostriatal but not in corticostriatal inputs. Both optogenetic and chemogenetic inhibition of the thalamostriatal pathway reverses motor deficits in parkinsonian mice [115] and hemiparkinsonian rats [116].

These studies support the notion of thalamic involvement in BG circuit dysregulation and in PD pathophysiology. In addition to providing new detailed information about the interplay between different brain circuits in the control of movement, the findings may offer a potential therapeutic approach in restoring normal motor behavior in PD.

Brainstem motor regions. The BG project down to the mesencephalic locomotor region (MLR), a brainstem region that controls locomotion. In mammals, it comprises the pedunculopontine nucleus (PPN) and the cuneiform nucleus. In humans, damage to the MLR is associated with locomotor deficits [117]. The MLR has been explored as a target for DBS to improve locomotor function in parkinsonian patients.
To investigate the locomotor function of MLR cell types and their control by BG circuitry, Roseberry et al. [118] combined cell-type-specific optogenetic manipulations, in vivo single-unit recording from identified cells, viralbased circuit mapping, and high-resolution behavioral assays to explore how signals from the BG control locomotion through the MLR. Their results highlighted the functional differences among cell types in the MLR and the specificity of BG-brainstem projections and demonstrated that the BG can initiate or suppress action by specific modulation of neuronal sub-types associated with a motor program.

The cholinergic PPN was reported as one of the brain structures with neuronal loss in PD patients [119]. Neurodegeneration of the PPN was also shown in an animal PD model based on proteasome inhibition [120]. Proteasome inhibition, discovered for the first time post mortem in the SNc of PD patients in 2001, successfully became an attractive model that mimics $P D$ in animals with a high degree of construct and expression, recapitulating almost all biochemical and behavioral PD features [121, 122]. Using transgenic rats that express Cre recombinase in cholinergic neurons (ChATCre), Pienaar et al. [123] showed that chemogenetic excitation of the rat PPN analogue, the nucleus tegmenti pedunculopontine (PPTg), reverses motor deficits in the $P D$ rat/mouse model based on unilateral intranigral proteasome inhibition by the irreversible proteasome inhibitor lactacystin. Interestingly, despite almost $40 \%$ neuronal loss in the PPTg of the lesioned hemisphere (compared to the intact hemisphere), DREADDsmediated cholinergic stimulation of the remaining neurons recovered or significantly improved motor performance, highlighting the therapeutic potential of PPN modulation in late phases of the disease.

\section{Mechanisms of deep brain stimulation treatment with optogenetics in Parkinson's disease}

Deep brain stimulation (DBS) is a therapeutic option for intractable neurological and psychiatric disorders, including PD and major depression. Because of the heterogeneity of brain tissues at the site of electrode placement, it has been challenging to elucidate the relevant target cell types and underlying mechanisms of DBS. Since STN is the target of DBS, it had been assumed that its action is on the nucleus itself. However, the therapeutic effects of DBS are accomplished by inducing fluctuation in the pathological neuronal activity over time, and the spatial specificity of the technique is still limited [124]. Optogenetic approaches present an opportunity to achieve cell-type-specific control with high temporal specificity on a large enough scale resolution to effectively repair network-level dysfunction.

Deisseroth and colleagues [32] demonstrated the potential for high-fidelity temporal control of individual neuronal spikes using an optogenetic approach. These initial exciting results have been followed by studies 
that sought to modulate circuits in freely behaving animals to further elucidate the pathophysiological role of specific brain structures and specific types of neurons. Models of PD were among the first to benefit from such an approach. Adopting the DBS protocol and using optogenetics to systematically drive or inhibit an array of distinct circuit elements in freely moving parkinsonian rodents, Gradinaru et al. [125] found that the therapeutic effects of DBS within the STN can be accounted for by direct selective stimulation of afferent axons projecting to this region. Surprisingly, neither direct NpHR-mediated optogenetic inhibition of the STN (via light-activated chloride pump), which reduced neuronal spiking in the STN by $80 \%$ nor high-frequency optogenetic stimulation (via channelrhodopsins light-activated cation channels, ChR2) delivered locally to the STN area, affected PD behavioral symptoms. Subsequent experiments revealed that similar motor improvement could also be achieved by optical stimulation of layer 5 projection neurons in the motor cortex, suggesting that the hyperdirect cortico-subthalamic pathway is critical in mediating the positive effects of stimulation on motor function in the rodent model of PD. Moreover, the effect of stimulation of afferent fibers to the STN was frequency-specific; optogenetic stimulation at $20 \mathrm{~Hz}$ worsened pathological motor activity, which is consistent with the reported role of exaggerated beta band oscillations in parkinsonian motor impairment.

Recently, Sanders and Jaeger [126] used a dual virus transfection technique that produces specific opsin expression in M1-STN projections to demonstrate that high frequency optogenetic stimulation $(100-130 \mathrm{~Hz})$ of cortico-subthalamic projections is sufficient to ameliorate bradykinesia in 6-OHDA-lesioned mice and to reduce pathological synchronization in the M1-STN pathway. In addition to orthodromic and antidromic effects, stimulation of cortico-subthalamic neurons may induce wide-spread increased glutamatergic activity due to collaterals that project to the thalamus and other brain regions in the motor-impaired hemisphere, thereby allowing increased locomotion. Given that the results were achieved through AAV injections in non-transgenic mice, it is possible that this approach may be applied to specific targeting of M1-STN projections in PD patients to ameliorate parkinsonian motor symptoms.

\section{Optogenetics and DREADDs \\ to explore the neural circuitry underlying levodopa-induced dyskinesia}

While dopamine replacement with levodopa may restore normal motor function, the induction of involuntary movements (LID) affects up to $80 \%$ of PD patients within 10-15 years of starting treatment, which significantly limits therapeutic efficacy. Aberrant activity in the striatum has been hypothesized to cause LID [127]. Until recently, LID pathology was largely presumed to reside within dMSNs and be associated with abnormal synaptic plasticity [128]. However, new data highlights the importance of functional coordination between iMSN and dMSN circuits and the complications that arise when the balance between the two is perturbed. Understanding the neural network mechanisms that form the basis of LID is essential for designing effective therapeutic strategies [129].

Alcacer et al. [130] recently reported the use of cell type-specific chemogenetics to investigate the role of dMSNs and iMSNs in models of parkinsonism and LID and revealed that direct and indirect pathways have opposing effects on whole-body movements and modulate both the therapeutic and dyskinetic responses to L-DOPA. Together, these results challenge the view that dMSN overactivity alone can explain LID, showing that fully-emerged dyskinesias can only be achieved by interventions that mimic the action of L-DOPA on both pathways.

A recent study by Girasole et al. [131] employed optogenetically labeled striatal single-unit recordings and targeted recombination in active populations (TRAP), a method to manipulate dyskinesia-associated neurons, to assess circuit dysfunction in experimental parkinsonism and LID and to establish a direct link between striatal activity and dyskinesia. They provided evidence that TRAPed cells are comprised of a specific subset of sensorimotor striatal neurons, predominantly from the direct pathway (dMSNs). Reactivation of TRAPed striatal neurons induces dyskinesia in the absence of levodopa. Conversely, inhibition of TRAPed cells, but not a nonspecific subset of direct pathway neurons, ameliorates LID. These results establish that a distinct subset of striatal neurons is causally involved in LID and indicate that successful therapeutic strategies for treating LID may require targeting functionally selective neuronal subtypes. In addition, in studies [74, 132] there was identified a subset of direct pathway neurons that exhibits levodopa-evoked abnormal increase in firing rates correlated with dyskinesia severity.

Several recent reports have implicated elevated striatal cholinergic neurotransmission in the motor complications induced by chronic L-DOPA therapy [133-137]. The induction of LIDs has been linked to enhanced ERK phosphorylation of striatal cholinergic interneurons (CINs) following repeated L-DOPA treatment, while selective ablation of CINs has been shown to attenuate LIDs in dyskinetic parkinsonian mice [133, 135]. Additional work by Aldrin-Kirk et al. [137], utilizing two transgenic Cre-driver rat lines, a choline acetyltransferase ChAT-Cre transgenic rat and a novel double-transgenic tyrosine hydroxylase TH-Cre/ChATCre rat, demonstrated that selective and reversible activation of CINs using chemogenetic (DREADD) receptors increases locomotor function in intact rats, potentiates the therapeutic effect of L-DOPA in rats with loss of nigral dopamine, and intensifies LIDs.

These findings provide key insights into the circuit mechanisms underlying parkinsonism and LID, with 
implications for developing targeted therapies that could be more precise and effective than levodopa alone.

\section{Chemogenetic applications in dopamine-cell replacement therapy}

The DREADD technique has been utilized in studies to evaluate new therapeutic approaches, such as cell replacement therapy. Currently available therapeutic strategies against PD (mostly pharmacological) are ineffective in altering disease progression and demonstrate only temporary alleviation of motor symptoms, with significant side effects. Therefore, strategies like cell grafting or viral gene transfer that aim to restore proper BG function via tissue regeneration are gaining popularity. Chemogenetic methodologies provide a powerful tool for remote modulation of graft activity, allowing for better understanding of molecular and systemic processes supporting transplant integration into host tissue and, more importantly, for temporary enhancement of motor performance in dopaminedepleted animal models. As seen in a series of studies in animal models of PD [138-141], cell grafting itself can enhance motor performance in dopamine-depleted animals (consistent with human studies [142]), while chemogenetics enables remote bimodal tuning of behavioral outputs depending on needs. Although many aspects of DREADD technology use in human graft modulation needs to be considered, its potential for translational application in the field of biomedicine is enormous.

\section{Conclusion}

Both optogenetics and DREADD methodology have greatly advanced our understanding of the relative contributions of distinct brain regions, celltypes, and individual neural circuit components to animal motor behavior in normal and disease states, such as Parkinson's disease. These approaches have conferred a previously unattainable level of spatiotemporal precision to the experimental design for monitoring and manipulating neuronal and circuit activity in PD animal models. Despite the complexity of the remodeling neural circuitry underlying progressive motor neurodegenerative disorders, the potency of the modulatory effects can be replicated between different species and with very different techniques. In addition, the ability to remotely modulate neural activity with these techniques minimizes the effect of stress-related factors on the outputs of sensitive behavioral tasks. Multiple studies provided evidence that interrogation of brain circuitry using optogenetic and chemogenetic approaches can successfully rescue motor impairments in animal models of PD, and an adjustable closed-loop design of DBS could be used to adaptively stimulate aberrant circuits. Optogenetics or chemogenetics can be combined with gene therapy and stem cell transfer, and DREADD methodology can provide a powerful tool for remote and non-invasive modulation of graft activity to support transplant integration into host tissue. Among the different viral vectors currently used for gene therapy in humans, AAV is reported to be safe for therapeutic application. All these technical developments hold promise for the use of optogenetics and pharmacogenetics in the clinical setting for the treatment of a variety of human diseases affecting the CNS.

Study funding. This work was supported by the Russian Foundation for Basic Research (grant number 18-015-00157-a) and the grant provided jointly by the Russian Foundation for Basic Research and the Ministry of Investments and Innovations of the Moscow Region (grant number 17-44-500312 p_a).

Conflicts of interest. The authors have no conflicts of interest to disclose.

\section{References}

1. Lees A.J., Hardy J., Revesz T. Parkinson's disease. Lancet 2009; 373(9680): 2055-2066, https://doi.org/10.1016/ s0140-6736(09)60492-x.

2. Pisani A., Bernardi G., Ding J., Surmeier D.J. Reemergence of striatal cholinergic interneurons in movement disorders. Trends Neurosci 2007; 30(10): 545-553, https://doi. org/10.1016/j.tins.2007.07.008.

3. Nakano K. Neural circuits and topographic organization of the basal ganglia and related regions. Brain Dev 2000; 22(Suppl 1): S5-S16, https://doi.org/10.1016/s0387-7604(00) 00139-x.

4. DeLong M.R., Wichmann T. Circuits and circuit disorders of the basal ganglia. Arch Neurol 2007; 64(1): 20, https://doi.org/10.1001/archneur.64.1.20.

5. Obeso J.A., Rodríguez-Oroz M.C., Benitez-Temino B., Blesa F.J., Guridi J., Marin C., Rodriguez M. Functional organization of the basal ganglia: therapeutic implications for Parkinson's disease. Mov Disord 2008; 23(Suppl 3): S548S559, https://doi.org/10.1002/mds.22062.

6. Wichmann T., DeLong M.R., Guridi J., Obeso J.A. Milestones in research on the pathophysiology of Parkinson's disease. Mov Disord 2011; 26(6): 1032-1041, https://doi. org/10.1002/mds.23695.

7. Gerfen C.R., Surmeier D.J. Modulation of striatal projection systems by dopamine. Annu Rev Neurosci 2011; 34(1): 441-466, https://doi.org/10.1146/annurev-neuro061010-113641.

8. Obeso J.A., Marin C., Rodriguez-Oroz C., Blesa J., Benitez-Temiño B., Mena-Segovia J., Rodríguez M., Olanow C.W. The basal ganglia in Parkinson's disease: current concepts and unexplained observations. Ann Neurol 2008; 64(Suppl 2): S30-S46, https://doi.org/10.1002/ana. 21481.

9. Albin R.L., Young A.B., Penney J.B. The functional anatomy of basal ganglia disorders. Trends Neurosci 1989; 12: 366-375, https://doi.org/10.1016/0166-2236(89)90074-x.

10. Maia T.V., Frank M.J. From reinforcement learning models to psychiatric and neurological disorders. Nat Neurosci 2011; 14(2): 154-162, https://doi.org/10.1038/nn.2723.

11. Cotzias G.C., Papavasiliou P.S., Gellene R. Modification of parkinsonism - chronic treatment with L-DOPA. N Engl 
J Med 1969; 13; 280(7): 337-345, https://doi.org/10.1056/ nejm196902132800701.

12. Marsden C.D. Parkinson's disease. Lancet 1990; 35(8695): 948-952, https://doi.org/10.1016/01406736(90)91006-v.

13. Nyholm D. The rationale for continuous dopaminergic stimulation in advanced Parkinson's disease. Parkinsonism Relat Disord 2007; 13(Suppl): S13-S17, https://doi.org/10. 1016/j.parkreldis.2007.06.005.

14. Dobrzanski G., Kossut M. Application of the DREADD technique in biomedical brain research. Pharmacol Rep 2017; 69(2): 213-221, https://doi.org/10.1016/j.pharep.2016.10.015.

15. Blandini F., Armentero M.T. Animal models of Parkinson's disease. FEBS J 2012; 279(7): 1156-1166, https:// doi.org/10.1111/j.1742-4658.2012.08491.x.

16. Bergman H., Feingold A., Nini A., Raz A., Slovin H., Abeles M., Vaadia E. Physiological aspects of information processing in the basal ganglia of normal and parkinsonian primates. Trends Neurosci 1998; 21: 32-38, https://doi. org/10.1016/s0166-2236(97)01151-x.

17. Bevan M.D., Magill P.J., Terman D., Bolam J.P., Wilson C.J. Move to the rhythm: oscillations in the subthalamic nucleus-external globus pallidus network. Trends Neurosci 2002; 25(10): 525-531, https://doi.org/10.1016/s01662236(02)02235-x.

18. Brown P. Oscillatory nature of human basal ganglia activity: relationship to the pathophysiology of Parkinson's disease. Mov Disord 2003; 18(4): 357-363, https://doi. org/10.1002/mds.10358.

19. Hutchison W.D., Dostrovsky J.O., Walters J.R., Courtemanche R., Boraud T., Goldberg J., Brown P. Neuronal oscillations in the basal ganglia and movement disorders: evidence from whole animal and human recordings. J Neurosci 2004; 24(42): 9240-9243, https://doi.org/10.1523/ jneurosci.3366-04.2004.

20. Gatev P., Darbin O., Wichmann T. Oscillations in the basal ganglia under normal conditions and in movement disorders. Mov Disord 2006; 21(10): 1566-1577, https://doi. org/10.1002/mds.21033.

21. Corbit V.L., Whalen T.C., Zitelli K.T., Crilly S.Y., Rubin J.E., Gittis A.H. Pallidostriatal projections promote $\beta$ oscillations in a dopamine-depleted biophysical network model. J Neurosci 2016; 36(20): 5556-5571, https://doi.org/10.1523/ jneurosci.0339-16.2016.

22. Leblois A., Meissner W., Bioulac B., Gross C.E., Hansel D., Boraud T. Late emergence of synchronized oscillatory activity in the pallidum during progressive parkinsonism. Eur J Neurosci 2007; 26(6): 1701-1713, https:// doi.org/10.1111/j.1460-9568.2007.05777.x.

23. Mallet N., Pogosyan A., Sharott A., Csicsvari J., Bolam J.P., Brown P., Magill P.J. Disrupted dopamine transmission and the emergence of exaggerated beta oscillations in subthalamic nucleus and cerebral cortex. J Neurosci 2008; 28(18): 4795-4806, https://doi.org/10.1523/ jneurosci.0123-08.2008.

24. Degos B., Deniau J.-M., Chavez M., Maurice N. Chronic but not acute dopaminergic transmission interruption promotes a progressive increase in cortical beta frequency synchronization: relationships to vigilance state and akinesia. Cereb Cortex 2009; 19(7): 1616-1630, https://doi.org/10.1093/ cercor/bhn199.

25. Kühn A.A., Kupsch A., Schneider G.H., Brown P. Reduction in subthalamic $8-35 \mathrm{~Hz}$ oscillatory activity correlates with clinical improvement in Parkinson's disease. Eur $J$ Neurosci 2006; 23(7): 1956-1960, https://doi.org/10.1111/ j.1460-9568.2006.04717.x.

26. Hammond C., Bergman H., Brown P. Pathological synchronization in Parkinson's disease: networks, models and treatments. Trends Neurosci 2007; 30(7): 357-364, https://doi. org/10.1016/j.tins.2007.05.004.

27. Kühn A.A., Tsui A., Aziz T., Ray N., Brücke C., Kupsch A., Schneider G.H., Brown P. Pathological synchronisation in the subthalamic nucleus of patients with Parkinson's disease relates to both bradykinesia and rigidity. Exp Neurol 2009; 215(2): 380-387, https://doi.org/10.1016/j. expneurol.2008.11.008.

28. Eusebio A., Thevathasan W., Doyle Gaynor L., Pogosyan A., Bye E., Foltynie T., Zrinzo L., Ashkan K., Aziz T., Brown P. Deep brain stimulation can suppress pathological synchronisation in parkinsonian patients. J Neurol Neurosurg Psychiatry 2011; 82(5): 569-573, https://doi.org/10.1136/ jnnp.2010.217489.

29. Little S., Pogosyan A., Kuhn A.A., Brown P. Beta band stability over time correlates with parkinsonian rigidity and bradykinesia. Exp Neurol 2012; 236(2): 383-388, https://doi. org/10.1016/j.expneurol.2012.04.024.

30. Oswal A., Beudel M., Zrinzo L., Limousin P., Hariz M., Foltynie T., Litvak V., Brown P. Deep brain stimulation modulates synchrony within spatially and spectrally distinct resting state networks in Parkinson's disease. Brain 2016; 139(Pt 5): 1482-1496, https://doi.org/10.1093/brain/aww048.

31. Armbruster B.N., Li X., Pausch M.H., Herlitze S., Roth B.L. Evolving the lock to fit the key to create a family of $G$ protein-coupled receptors potently activated by an inert ligand. Proc Natl Acad Sci U S A 2007; 104(12): 5163-5168, https:// doi.org/10.1073/pnas.0700293104.

32. Boyden E.S., Zhang F., Bamberg E., Nagel G., Deisseroth K. Millisecond-timescale, genetically targeted optical control of neural activity. Nat Neurosci 2005; 8(9): 1263-1268, https://doi.org/10.1038/nn1525.

33. Vazey E.M., Aston-Jones G. New tricks for old dogmas: optogenetic and designer receptor insights for Parkinson's disease. Brain Res 2013; 1511: 153-163, https://doi. org/10.1016/j.brainres.2013.01.021.

34. Wiegert J.S., Mahn M., Prigge M., Printz Y., Yizhar O. Silencing neurons: tools, applications, and experimental constraints. Neuron 2017; 95(3): 504-529, https://doi. org/10.1016/j.neuron.2017.06.050.

35. Fenno L., Yizhar O., Deisseroth K. The development and application of optogenetics. Annu Rev Neurosci 2011; 34(1): 389-412, https://doi.org/10.1146/annurevneuro-061010-113817.

36. Nagel G., Szellas T., Kateriya S., Adeishvili N., Hegemann P., Bamberg E. Channelrhodopsins: directly lightgated cation channels. Biochem Soc Trans 2005; 33(4): 863866, https://doi.org/10.1042/bst0330863.

37. Chow B.Y., Han X., Dobry A.S., Qian X., Chuong A.S., Li M., Henninger M.A., Belfort G.M., Lin Y., Monahan P.E., Boyden E.S. High-performance genetically targetable optical neural silencing by light-driven proton pumps. Nature 2010; 463(7277): 98-102, https://doi.org/10.1038/nature08652.

38. Gradinaru V., Thompson K.R., Deisseroth K. eNpHR: a natronomonas halorhodopsin enhanced for optogenetic applications. Brain Cell Biol 2008; 36(1-4): 129-139, https:// doi.org/10.1007/s11068-008-9027-6.

39. Farrell M.S., Roth B.L. Pharmacosynthetics: 
reimagining the pharmacogenetic approach. Brain Res 2013; 1511: 6-20, https://doi.org/10.1016/j.brainres.2012.09.043.

40. Sternson S.M., Roth B.L. Chemogenetic tools to interrogate brain functions. Annu Rev Neurosci 2014; 37: 387407, https://doi.org/10.1146/annurev-neuro-071013-014048.

41. Vardy E., Robinson J.E., Li C., Olsen R.H.J., DiBerto J.F., Giguere P.M., Sassano F.M., Huang X.P., Zhu H., Urban D.J., White K.L., Rittiner J.E., Crowley N.A., Pleil K.E., Mazzone C.M., Mosier P.D., Song J., Kash T.L., Malanga C.J., Krashes M.J., Roth B.L. A new DREADD facilitates the multiplexed chemogenetic interrogation of behavior. Neuron 2015; 86(4): 936-946, https://doi.org/10.1016/j.neuron.2015.03.065.

42. Lee H.M., Giguere P.M., Roth B.L. DREADDs: novel tools for drug discovery and development. Drug Discov Today 2014; 19(4): 469-473, https://doi.org/10.1016/j. drudis.2013.10.018.

43. Zhu H., Roth B.L. Silencing synapses with DREADDs. Neuron 2014; 82(4): 723-725, https://doi.org/10.1016/j. neuron.2014.05.002.

44. Urban D.J., Roth B.L. DREADDs (designer receptors exclusively activated by designer drugs): chemogenetic tools with therapeutic utility. Annu Rev Pharmacol Toxicol 2015; 55(1): 399-417, https://doi.org/10.1146/annurevpharmtox-010814-124803.

45. Roth B.L. DREADDs for neuroscientists. Neuron 2016; 89(4): 683-694, https://doi.org/10.1016/j.neuron.2016.01.040.

46. Bernstein J.G., Boyden E.S. Optogenetic tools for analyzing the neural circuits of behavior. Trends Cogn Sci 2011; 15(12): 592-600, https://doi.org/10.1016/j.tics.2011.10.003.

47. Sjulson L., Cassataro D., DasGupta S., Miesenböck G. Cell-specific targeting of genetically encoded tools for neuroscience. Annu Rev Genet 2016; 50(1): 571-594, https:// doi.org/10.1146/annurev-genet-120215-035011.

48. Jiang J., Cui H., Rahmouni K. Optogenetics and pharmacogenetics: principles and applications. Am J Physiol Regul Integr Comp Physiol 2017; 313(6): R633-R645, https:// doi.org/10.1152/ajpregu.00091.2017.

49. Boyden E.S. A history of optogenetics: the development of tools for controlling brain circuits with light. F1000 Biol Rep 2011; 3: 11, https://doi.org/10.3410/b3-11.

50. Deisseroth K. Optogenetics: 10 years of microbial opsins in neuroscience. Nat Neurosci 2015; 18(9): 1213-1225, https://doi.org/10.1038/nn.4091.

51. Rajasethupathy P., Ferenczi E., Deisseroth K. Targeting neural circuits. Cell 2016; 165(3): 524-534, https://doi. org/10.1016/j.cell.2016.03.047.

52. Cui G., Jun S.B., Jin X., Pham M.D., Vogel S.S., Lovinger D.M., Costa R.M. Concurrent activation of striatal direct and indirect pathways during action initiation. Nature 2013; 494(7436): 238-242, https://doi.org/10.1038/ nature11846.

53. Freeze B.S., Kravitz A.V., Hammack N., Berke J.D., Kreitzer A.C. Control of basal ganglia output by direct and indirect pathway projection neurons. J Neurosci 2013; 33(47): 18531-18539, https://doi.org/10.1523/jneurosci.1278-13.2013.

54. Lenz J.D., Lobo M.K. Optogenetic insights into striatal function and behavior. Behav Brain Res 2013; 255: 44-54, https://doi.org/10.1016/j.bbr.2013.04.018.

55. Kravitz A.V., Freeze B.S., Parker P.R., Kay K., Thwin M.T., Deisseroth K., Kreitzer A.C. Regulation of parkinsonian motor behaviours by optogenetic control of basal ganglia circuitry. Nature 2010; 466(7306): 622-666, https://doi. org/10.1038/nature09159.
56. Kravitz A.V., Tye L.D., Kreitzer A.C. Distinct roles for direct and indirect pathway striatal neurons in reinforcement. Nat Neurosci 2012; 15(6): 816-818, https://doi.org/10.1038/ nn.3100.

57. Lee H.J., Weitz A.J., Bernal-Casas D., Duffy B.A., Choy M., Kravitz A.V., Kreitzer A.C., Lee J.H. Activation of direct and indirect pathway medium spiny neurons drives distinct brain-wide responses. Neuron 2016; 91(2): 412-424, https://doi.org/10.1016/j.neuron.2016.06.010.

58. Oldenburg I.A., Sabatini B.L. Antagonistic but not symmetric regulation of primary motor cortex by basal ganglia direct and indirect pathways. Neuron 2015; 86(5): 1174-1181, https://doi.org/10.1016/j.neuron.2015.05.008.

59. Tecuapetla F., Matias S., Dugue G.P., Mainen Z.F., Costa R.M. Balanced activity in basal ganglia projection pathways is critical for contraversive movements. Nat Commun 2014; 5(1), https://doi.org/10.1038/ncomms5315.

60. Tecuapetla F., Jin X., Lima S.Q., Costa R.M. Complementary contributions of striatal projection pathways to action initiation and execution. Cell 2016; 166(3): 703-715, https://doi.org/10.1016/j.cell.2016.06.032.

61. Glajch K.E., Kelver D.A., Hegeman D.J., Cui Q., Xenias H.S., Augustine E.C., Hernández V.M., Verma N., Huang T.Y., Luo M., Justice N.J., Chan C.S. Npas1+ pallidal neurons target striatal projection neurons. J Neurosci 2016; 36(20): 5472-5488, https://doi.org/10.1523/ jneurosci.1720-15.2016.

62. Saunders A., Huang K.W., Sabatini B.L. Globus pallidus externus neurons expressing parvalbumin interconnect the subthalamic nucleus and striatal interneurons. PLoS One 2016; 11(2): e0149798, https://doi.org/10.1371/journal.pone.0149798.

63. Straub C., Saulnier J.L., Bègue A., Feng D.D., Huang K.W., Sabatini B.L. Principles of synaptic organization of GABAergic interneurons in the striatum. Neuron 2016; 92(1): 84-92, https://doi.org/10.1016/j.neuron.2016.09.007.

64. Tritsch N.X., Ding J.B., Sabatini B.L. Dopaminergic neurons inhibit striatal output through non-canonical release of GABA. Nature 2012; 490(7419): 262-266, https://doi. org/10.1038/nature11466.

65. Straub C., Tritsch N.X., Hagan N.A., Gu C., Sabatini B.L. Multiphasic modulation of cholinergic interneurons by nigrostriatal afferents. J Neurosci 2014; 34(25): 8557-8569, https://doi.org/10.1523/jneurosci.0589-14.2014.

66. Howe M.W., Dombeck D.A. Rapid signalling in distinct dopaminergic axons during locomotion and reward. Nature 2016; 535(7613): 505-510, https://doi.org/10.1038/nature18942.

67. Miguelez C., Morin S., Martinez A., Goillandeau M., Bezard E., Bioulac B., Baufreton J. Altered pallido-pallidal synaptic transmission leads to aberrant firing of globus pallidus neurons in a rat model of Parkinson's disease. J Physiol 2012; 590(22): 5861-5875, https://doi.org/10.1113/ jphysiol.2012.241331.

68. Fieblinger T., Graves S.M., Sebel L.E., Alcacer C., Plotkin J.L., Gertler T.S., Chan C.S., Heiman M., Greengard P., Cenci M.A., Surmeier D.J. Cell type-specific plasticity of striatal projection neurons in parkinsonism and L-DOPA-induced dyskinesia. Nat Commun 2014; 5(1), https://doi.org/10.1038/ ncomms6316.

69. Chu H.-Y., Atherton J.F., Wokosin D., Surmeier D.J., Bevan M.D. Heterosynaptic regulation of external globus pallidus inputs to the subthalamic nucleus by the motor cortex. Neuron 2015; 85(2): 364-376, https://doi.org/10.1016/j. neuron.2014.12.022. 
70. Alexander G.E., Crutcher M.D. Functional architecture of basal ganglia circuits: neural substrates of parallel processing. Trends Neurosci 1990; 13(7): 266-271, https://doi. org/10.1016/0166-2236(90)90107-I.

71. DeLong M.R. Primate models of movement disorders of basal ganglia origin. Trends Neurosci 1990; 13(7): 281-285, https://doi.org/10.1016/0166-2236(90)90110-v.

72. Gerfen C.R., Engber T.M., Mahan L.C., Susel Z., Chase T.N., Monsma F.J. Jr., Sibley D.R. D1Rs and D2Rs dopamine receptor-regulated gene expression of striatonigral and striatopallidal neurons. Science 1990; 250(4986): 14291432, https://doi.org/10.1126/science.2147780.

73. Durieux P.F., Schiffmann S.N., de Kerchove d'Exaerde A. Differential regulation of motor control and response to dopaminergic drugs by D1RsR and D2RsR neurons in distinct dorsal striatum subregions. EMBO J 2011; 31(3): 640-653, https://doi.org/10.1038/emboj.2011.400.

74. Ryan M.B., Bair-Marshall C., Nelson A.B. Aberrant striatal activity in parkinsonism and levodopa-induced dyskinesia. Cell Rep 2018; 23(12): 3438-3446.e5, https://doi. org/10.1016/j.celrep.2018.05.059.

75. Tai L.-H., Lee A.M., Benavidez N., Bonci A., Wilbrecht L. Transient stimulation of distinct subpopulations of striatal neurons mimics changes in action value. Nat Neurosci 2012; 15(9): 1281-1289, https://doi.org/10.1038/nn.3188.

76. Isomura Y., Takekawa T., Harukuni R., Handa T., Aizawa H., Takada M., Fukai T. Reward-modulated motor information in identified striatum neurons. I Neurosci 2013; 33(25): 10209-10220, https://doi.org/10.1523/ jneurosci.0381-13.2013.

77. Jin X., Tecuapetla F., Costa R.M. Basal ganglia subcircuits distinctively encode the parsing and concatenation of action sequences. Nat Neurosci 2014; 17(3): 423-430, https://doi.org/10.1038/nn.3632.

78. Tubert C., Taravini I.R.E., Flores-Barrera E., Sánchez G.M., Prost M.A., Avale M.E., Tseng K.Y., Rela L., Murer M.G. Decrease of a current mediated by Kv 1.3 channels causes striatal cholinergic interneuron hyperexcitability in experimental parkinsonism. Cell Rep 2016; 16(10): 27492762, https://doi.org/10.1016/j.celrep.2016.08.016.

79. Maurice N,, Liberge M., Jaouen F., Ztaou S., Hanini M., Camon J., Deisseroth K., Amalric M., Kerkerian-Le Goff L., Beurrier C. Striatal cholinergic interneurons control motor behavior and basal ganglia function in experimental parkinsonism. Cell Rep 2015; 13(4): 657-666, https://doi. org/10.1016/j.celrep.2015.09.034.

80. DeLong M.R., Wichmann T. Basal ganglia circuits as targets for neuromodulation in parkinson disease. JAMA Neurol 2015; 72(11): 1354, https://doi.org/10.1001/ jamaneurol.2015.2397.

81. Mastro K.J., Zitelli K.T., Willard A.M., Leblanc K.H., Kravitz A.V., Gittis A.H. Cell-specific pallidal intervention induces long-lasting motor recovery in dopamine-depleted mice. Nat Neurosci 2017; 20(6): 815-823, https://doi. org/10.1038/nn.4559.

82. Assaf F., Schiller Y. A chemogenetic approach for treating experimental Parkinson's disease. Mov Disord 2019; 34(4): 469-479, https://doi.org/10.1002/mds.27554.

83. Lanciego J.L., Luquin N., Obeso J.A. Functional neuroanatomy of the basal ganglia. Cold Spring Harb Perspect Med 2012; 2(12): a009621-a009621, https://doi.org/10.1101/ cshperspect.a009621.

84. Galvan A., Devergnas A., Wichmann T. Alterations in neuronal activity in basal ganglia-thalamocortical circuits in the parkinsonian state. Front Neuroanat 2015; 9: 5, https://doi. org/10.3389/fnana.2015.00005.

85. Przedborski S. The two-century journey of Parkinson disease research. Nat Rev Neurosci 2017; 18(4): 251-259, https://doi.org/10.1038/nrn.2017.25.

86. Moon H.C., Won S.Y., Kim E.G., Kim H.K., Cho C.B., Park Y.S. Effect of optogenetic modulation on entopeduncular input affects thalamic discharge and behavior in an AAV2$\alpha$-synuclein-induced hemiparkinson rat model. Neurosci Lett 2018; 662: 129-135, https://doi.org/10.1016/j. neulet.2017.10.019.

87. Kötter R. Postsynaptic integration of glutamatergic and dopaminergic signals in the striatum. Prog Neurobiol 1994; 44(2): 163-196, https://doi.org/10.1016/03010082(94)90037-x.

88. Fernandez E., Schiappa R., Girault J.-A., Le Novère N. DARPP-32 is a robust integrator of dopamine and glutamate signals. PLoS Comput Biol 2006; 2(12): e176, https://doi. org/10.1371/journal.pcbi.0020176.eor.

89. Hunnicutt B.J., Jongbloets B.C., Birdsong W.T., Gertz K.J., Zhong H., Mao T. A comprehensive excitatory input map of the striatum reveals novel functional organization. eLife 2016; 5, https://doi.org/10.7554/elife.19103.

90. Marinelli L., Quartarone A., Hallett M., Frazzitta G., Ghilardi M.F. The many facets of motor learning and their relevance for Parkinson's disease. Clin Neurophysiol 2017; 128(7): 1127-1141, https://doi.org/10.1016/j.clinph. 2017.03.042.

91. Gruszka A., Hampshire A., Barker R.A., Owen A.M. Normal aging and Parkinson's disease are associated with the functional decline of distinct frontal-striatal circuits. Cortex 2017; 93: 178-192, https://doi.org/10.1016/j. cortex.2017.05.020.

92. Churchland M.M., Cunningham J.P., Kaufman M.T., Foster J.D., Nuyujukian P., Ryu S.I., Shenoy K.V. Neural population dynamics during reaching. Nature 2012; 487(7405): 51-56, https://doi.org/10.1038/nature11129.

93. Omrani M., Kaufman M.T., Hatsopoulos N.G., Cheney P.D. Perspectives on classical controversies about the motor cortex. J Neurophysiol 2017; 118(3): 1828-1848, https:// doi.org/10.1152/jn.00795.2016.

94. Rothwell P.E., Hayton S.J., Sun G.L., Fuccillo M.V., Lim B.K., Malenka R.C. Input- and output-specific regulation of serial order performance by corticostriatal circuits. Neuron 2015; 88(2): 345-356, https://doi.org/10.1016/j. neuron.2015.09.035.

95. Obeso J.A., Rodriguez-Oroz M.C., Rodriguez M., Lanciego J.L., Artieda J., Gonzalo N., Olanow C.W. Pathophysiology of the basal ganglia in Parkinson's disease. Trends Neurosci 2000; 23: S8-S19, https://doi.org/10.1016/ s1471-1931(00)00028-8.

96. Litvak V., Jha A., Eusebio A., Oostenveld R., Foltynie T., Limousin P., Zrinzo L., Hariz M.I., Friston K., Brown P. Resting oscillatory cortico-subthalamic connectivity in patients with Parkinson's disease. Brain 2010; 134(2): 359-374, https://doi. org/10.1093/brain/awq332.

97. Whitmer D., de Solages C., Hill B., Yu H., Henderson J.M., Bronte-Stewart H. High frequency deep brain stimulation attenuates subthalamic and cortical rhythms in Parkinson's disease. Front Hum Neurosci 2012; 6:155, https:// doi.org/10.3389/fnhum.2012.00155.

98. de Hemptinne C., Ryapolova-Webb E.S., Air E.L., 
Garcia P.A., Miller K.J., Ojemann J.G., Ostrem J.L., Galifianakis N.B., Starr P.A. Exaggerated phase-amplitude coupling in the primary motor cortex in Parkinson disease. Proc Natl Acad Sci U S A 2013; 110(12): 4780-4785, https:// doi.org/10.1073/pnas.1214546110.

99. Shimamoto S.A., Ryapolova-Webb E.S., Ostrem J.L., Galifianakis N.B., Miller K.J., Starr P.A. Subthalamic nucleus neurons are synchronized to primary motor cortex local field potentials in Parkinson's disease. J Neurosci 2013; 33(17): 7220-7233, https://doi.org/10.1523/jneurosci.467612.2013 .

100. Sharott A., Gulberti A., Zittel S., Tudor Jones A.A., Fickel U., Münchau A., Köppen J.A., Gerloff C., Westphal M., Buhmann C., Hamel W., Engel A.K., Moll C.K. Activity parameters of subthalamic nucleus neurons selectively predict motor symptom severity in Parkinson's disease. J Neurosci 2014; 34(18): 6273-6285, https://doi.org/10.1523/ jneurosci.1803-13.2014.

101. Delaville C., McCoy A.J., Gerber C.M., Cruz A.V., Walters J.R. Subthalamic nucleus activity in the awake hemiparkinsonian rat: relationships with motor and cognitive networks. J Neurosci 2015; 35(17): 6918-6930, https://doi. org/10.1523/jneurosci.0587-15.2015.

102. Mathai A., Ma Y., Paré J.-F., Villalba R.M., Wichmann T., Smith Y. Reduced cortical innervation of the subthalamic nucleus in MPTP-treated parkinsonian monkeys. Brain 2015; 138(4): 946-962, https://doi.org/10.1093/brain/ awv018.

103. Chu H.-Y., Mclver E.L., Kovaleski R.F., Atherton J.F., Bevan M.D. Loss of Hyperdirect pathway cortico-subthalamic inputs following degeneration of midbrain dopamine neurons. Neuron 2017; 95(6): 1306-1318.e5, https://doi.org/10.1016/j. neuron.2017.08.038.

104. Kim J., Kim D. Rebound excitability mediates motor abnormalities in Parkinson's disease. BMB Rep 2018; 51(1): 3-4, https://doi.org/10.5483/bmbrep.2018.51.1.004.

105. Yasukawa T., Kita T., Xue Y., Kita H. Rat intralaminar thalamic nuclei projections to the globus pallidus: a biotinylated dextran amine anterograde tracing study. J Comp Neurol 2004; 471(2): 153-167, https://doi.org/10.1002/cne.20029.

106. Alloway K.D., Smith J.B., Watson G.D.R. Thalamostriatal projections from the medial posterior and parafascicular nuclei have distinct topographic and physiologic properties. J Neurophysiol 2014; 111(1): 36-50, https://doi. org/10.1152/jn.00399.2013.

107. Kita T., Shigematsu N., Kita H. Intralaminar and tectal projections to the subthalamus in the rat. Eur J Neurosci 2016; 44(11): 2899-2908, https://doi.org/10.1111/ejn.13413.

108. Henderson J.M., Carpenter K., Cartwright H., Halliday G.M. Degeneration of the centré median-parafascicular complex in Parkinson's disease. Ann Neurol 2000; 47(3): 345352, https://doi.org/10.1002/1531-8249(200003)47:3<345::aidana10>3.3.co;2-m.

109. Villalba R.M., Wichmann T., Smith Y. Neuronal loss in the caudal intralaminar thalamic nuclei in a primate model of Parkinson's disease. Brain Struct Funct 2013; 219(1): 381394, https://doi.org/10.1007/s00429-013-0507-9.

110. Peppe A., Gasbarra A., Stefani A., Chiavalon C., Pierantozzi M., Fermi E., Stanzione P., Caltagirone C., Mazzone P. Deep brain stimulation of CM/PF of thalamus could be the new elective target for tremor in advanced Parkinson's disease? Parkinsonism Relat Disord 2008; 14(6): 501-504, https://doi.org/10.1016/j.parkreldis.2007.11.005.
111. Jouve L., Salin P., Melon C., Kerkerian-Le Goff L. Deep brain stimulation of the center median-parafascicular complex of the thalamus has efficient anti-parkinsonian action associated with widespread cellular responses in the basal ganglia network in a rat model of Parkinson's disease. J Neurosci 2010; 30(29): 9919-9928, https://doi.org/10.1523/ jneurosci.1404-10.2010.

112. Yan W., Zhang Q.J., Liu J., Wang T., Wang S., Liu X., Chen L., Gui Z.H. The neuronal activity of thalamic parafascicular nucleus is conversely regulated by nigrostriatal pathway and pedunculopontine nucleus in the rat. Brain Res 2008; 1240: 204-212, https://doi.org/10.1016/j.brainres.2008.09.015.

113. Nevado-Holgado A.J., Mallet N., Magill P.J., Bogacz R. Effective connectivity of the subthalamic nucleusglobus pallidus network during Parkinsonian oscillations. J Physiol 2014; 592(7): 1429-1455, https://doi.org/10.1113/ jphysiol.2013.259721.

114. Smith Y., Galvan A., Ellender T.J., Doig N., Villalba R.M., Huerta-Ocampo I., Wichmann T., Bolam J.P. The thalamostriatal system in normal and diseased states. Front Syst Neurosci 2014; 8: 5, https://doi.org/10.3389/fnsys.2014.00005.

115. Parker P.R.L., Lalive A.L., Kreitzer A.C. Pathwayspecific remodeling of thalamostriatal synapses in parkinsonian mice. Neuron 2016; 89(4): 734-740, https://doi.org/10.1016/j. neuron.2015.12.038.

116. Brazhnik E., Novikov N., Preston M.W., Weiss A.R.Jr., McCoy A.J., Walters J.R. Effects of inhibitory DREADD activation in the parafascicular thalamic nucleus on nigral and cortical high beta oscillations and motor behavior in hemiparkinsonian rats. SFN Abstract; 2018.

117. Kuo S.-H., Kenney C., Jankovic J. Bilateral pedunculopontine nuclei strokes presenting as freezing of gait. Mov Disord 2008; 23(4): 616-619, https://doi.org/10.1002/ mds. 21917.

118. Roseberry T.K., Lee A.M., Lalive A.L., Wilbrecht L., Bonci A., Kreitzer A.C. Cell-type-specific control of brainstem locomotor circuits by basal ganglia. Cell 2016; 164(3): 526537, https://doi.org/10.1016/j.cell.2015.12.037.

119. Hirsch E.C., Graybiel A.M., Duyckaerts C., JavoyAgid F. Neuronal loss in the pedunculopontine tegmental nucleus in Parkinson disease and in progressive supranuclear palsy. Proc Natl Acad Sci U S A 1987; 84(16): 5976-5980, https://doi.org/10.1073/pnas.84.16.5976.

120. Pienaar I.S., Harrison I.F., Elson J.L., Bury A., Woll P., Simon A.K., Dexter D.T. An animal model mimicking pedunculopontine nucleus cholinergic degeneration in Parkinson's disease. Brain Struct Funct 2013; 220(1): 479500, https://doi.org/10.1007/s00429-013-0669-5.

121. McNaught K.S., Jenner P. Proteasomal function is impaired in substantia nigra in Parkinson's disease. Neurosci Lett 2001; 297(3): 191-194, https://doi.org/10.1016/s03043940(00)01701-8.

122. McNaught K.S., Björklund L.M., Belizaire R., Isacson O., Jenner P., Olanow C.W. Proteasome inhibition causes nigral degeneration with inclusion bodies in rats. Neuroreport 2002; 13(11): 1437-1441, https://doi. org/10.1097/00001756-200208070-00018.

123. Pienaar I.S., Gartside S.E., Sharma P., De Paola V., Gretenkord S., Withers D., Elson J.L., Dexter D.T. Pharmacogenetic stimulation of cholinergic pedunculopontine neurons reverses motor deficits in a rat model of Parkinson's disease. Mol Neurodegener 2015; 10(1): 47, https://doi. org/10.1186/s13024-015-0044-5. 
124. Little S., Brown P. Focusing brain therapeutic interventions in space and time for Parkinson's disease. Curr Biol 2014; 24(18): R898-R909, https://doi.org/10.1016/j. cub.2014.08.002.

125. Gradinaru V., Mogri M., Thompson K.R., Henderson J.M., Deisseroth K. Optical deconstruction of parkinsonian neural circuitry. Science 2009; 324(5925): 354359, https://doi.org/10.1126/science.1167093.

126. Sanders T.H., Jaeger D. Optogenetic stimulation of cortico-subthalamic projections is sufficient to ameliorate bradykinesia in 6-ohda lesioned mice. Neurobiol Dis 2016; 95 : 225-237, https://doi.org/10.1016/j.nbd.2016.07.021.

127. Jenner P. Molecular mechanisms of L-DOPA-induced dyskinesia. Nat Rev Neurosci 2008; 9(9): 665-677, https://doi. org/10.1038/nrn2471.

128. Suarez L.M., Solis O., Aguado C., Lujan R., Moratalla R. L-DOPA oppositely regulates synaptic strength and spine morphology in D1Rs and D2Rs striatal projection neurons in dyskinesia. Cereb Cortex 2016; 26(11): 4253-4264, https://doi.org/10.1093/cercor/bhw263.

129. Cenci M.A., Jörntell H., Petersson P. On the neuronal circuitry mediating L-DOPA-induced dyskinesia. I Neural Transm 2018; 125(8): 1157-1169, https://doi.org/10.1007/ s00702-018-1886-0.

130. Alcacer C., Andreoli L., Sebastianutto I., Jakobsson J., Fieblinger T., Cenci M.A. Chemogenetic stimulation of striatal projection neurons modulates responses to Parkinson's disease therapy. J Clin Invest 2017; 127(2): 720-734, https://doi.org/10.1172/jci90132.

131. Girasole A.E., Lum M.Y., Nathaniel D., BairMarshall C.J., Guenthner C.J., Luo L., Kreitzer A.C., Nelson A.B. A subpopulation of striatal neurons mediates levodopa-induced dyskinesia. Neuron 2018; 97(4): 787-795.e6, https://doi.org/10.1016/j.neuron.2018.01.017.

132. Sagot B., Li L., Zhou F.-M. Hyperactive response of direct pathway striatal projection neurons to L-DOPA and D1 agonism in freely moving parkinsonian mice. Front Neural Circuits 2018; 12: 57, https://doi.org10.3389/fncir.2018.00057.

133. Ding Y., Won L., Britt J.P., Lim S.A.O., McGehee D.S., Kang U.J. Enhanced striatal cholinergic neuronal activity mediates L-DOPA-induced dyskinesia in parkinsonian mice. Proc Natl Acad Sci U S A 2010; 108(2): 840-845, https://doi. org/10.1073/pnas.1006511108.

134. Lim S.A.O., Kang U.J., McGehee D.S. Striatal cholinergic interneuron regulation and circuit effects. Front Synaptic Neurosci 2014; 6, https://doi.org/10.3389/ fnsyn.2014.00022.

135. Won L., Ding Y., Singh P., Kang U.J. Striatal cholinergic cell ablation attenuates L-DOPA induced dyskinesia in parkinsonian mice. J Neurosci 2014; 34(8): 3090-3094, https://doi.org/10.1523/jneurosci.2888-13.2014.

136. Shen W., Plotkin J.L., Francardo V., Ko W.K., Xie Z., Li Q., Fieblinger T., Wess J., Neubig R.R., Lindsley C.W., Conn P.J., Greengard P., Bezard E., Cenci M.A., Surmeier D.J. M4 muscarinic receptor signaling ameliorates striatal plasticity deficits in models of L-DOPA-induced dyskinesia. Neuron 2015; 88(4): 762-773, https://doi.org/10.1016/j.neuron.2015.10.039.

137. Aldrin-Kirk P., Heuer A., Rylander Ottosson D., Davidsson M., Mattsson B., Björklund T. Chemogenetic modulation of cholinergic interneurons reveals their regulating role on the direct and indirect output pathways from the striatum. Neurobiol Dis 2018; 109: 148-162, https://doi. org/10.1016/j.nbd.2017.10.010.

138. Dell'Anno M.T., Caiazzo M., Leo D., Dvoretskova E., Medrihan L., Colasante G., Giannelli S., Theka I., Russo G., Mus L., Pezzoli G., Gainetdinov R.R., Benfenati F., Taverna S., Dityatev A., Broccoli V. Remote control of induced dopaminergic neurons in parkinsonian rats. J Clin Invest 2014; 124(7): 3215-3229, https://doi.org/10.1172/jci74664.

139. Aldrin-Kirk P., Heuer A., Wang G., Mattsson B., Lundblad M., Parmar M., Björklund T. DREADD modulation of transplanted DA neurons reveals a novel parkinsonian dyskinesia mechanism mediated by the Serotonin 5-HT6 receptor. Neuron 2016; 90(5): 955-968, https://doi. org/10.1016/j.neuron.2016.04.017.

140. Chen Y., Xiong M., Dong Y., Haberman A., Cao J., Liu H., Zhou W., Zhang S.C. Chemical control of grafted human PSC-derived neurons in a mouse model of Parkinson's disease. Cell Stem Cell 2016; 18(6): 817-826, https://doi. org/10.1227/01.neu.0000499710.89208.c.

141. Zuo F., Xiong F., Wang X., Li X., Wang R., Ge W., Bao $X$. Intrastriatal transplantation of human neural stem cells restores the impaired subventricular zone in parkinsonian mice. Stem Cells 2017; 35(6): 1519-1531, https://doi.org/10.1002/ stem.2616.

142. Politis M., Lindvall O. Clinical application of stem cell therapy in Parkinson's disease. BMC Med 2012; 10: 1, https:// doi.org/10.1186/1741-7015-10-1. 